\title{
Multi-dimensional expansion of urban space through the lens of land use: The case study of Nanjing City, China
}

\author{
QIAO Weifeng ${ }^{1,2,4}$, GAO Junbo ${ }^{2,3},{ }^{*}$ GUO Yuanzhi ${ }^{2,5}, \mathrm{JI}$ Qingqing ${ }^{1}$, WU Ju${ }^{1}$, \\ $\mathrm{CAO} \mathrm{Min}^{1,4}$
}

1. School of Geography, Nanjing Normal University, Nanjing 210023, China;

2. Institute of Geographic Sciences and Natural Resources Research, CAS, Beijing 100101, China;

3. School of Geographic Sciences, Xinyang Normal University, Xinyang 464000, Henan, China;

4. Jiangsu Center for Collaborative Innovation in Geographical Information Resource Development and Application, Nanjing 210023, China;

5. University of Chinese Academy of Sciences, Beijing 100049, China

\begin{abstract}
The study of multi-dimensional expansion of urban space (MEUS) addresses the laws of urban spatial expansion from all directions and angles. Using Nanjing as an example, this paper constructs multi-temporal, urban three-dimensional models based on RS and GIS technology and then conducts qualitative and quantitative analysis of MEUS using plot ratio change maps and MEUS quantitative index for built-up areas. Based especially on the concept of volume growth contribution rate, this paper analyzes the characteristics of MEUS in different stages. The results show that in 2000-2004, planar expansion played the main role, the internal potential development (IPD) intensity of the urban built-up areas was relatively large, and the volume growth contribution rate was low; in 2004-2008, planar expansion accelerated, and IPD slowed down; in 2008-2012, planar expansion slowed while IPD intensity increased; the contribution rates of volume growth of urban IPD for the three periods were $22.21 \%, 24.51 \%$ and $73.38 \%$, respectively. This study expands the research perspective of urban spatial expansion, and the adopted methods are instructive and meaningful for MEUS research. In addition, the results of this study will deepen the understanding of MEUS laws and help improve scientific decision-making for urban planning and urban land use management.
\end{abstract}

Keywords: urban land use; plot ratio change map; multi-dimensional expansion; volume growth contribution rate; Nanjing

Received: 2018-07-11 Accepted: 2018-12-19

Foundation: National Natural Science Foundation of China, No.41871178, No.41671385, No.41371172

Author: Qiao Weifeng (1975-), PhD and Associate Professor, specialized in land use, urban-rural development and the application of GIS and remote sensing. E-mail: qiaoweifeng@njnu.edu.cn

"Corresponding author: Guo Yuanzhi, PhD Candidate, E-mail: guoyz.16b@igsnrr.ac.cn 


\section{Introduction}

At present, urbanization is accelerating globally (UN, 2007; Liu and Li, 2017). Urban spatial expansion, as an important aspect of the urbanization process, has always been a hot topic globally in the fields of urban geography, urban planning and urban land use research (Anas, 1976; Liu et al., 2016; Wen et al., 2016). China entered a period of rapid urbanization from the 1990s (Chi et al., 2015); a large number of cities have expanded rapidly, with city sizes reaching the largest scale ever in human history (Wang et al., 2012; Zhao et al., 2012), and will continue to expand. Many scholars have analyzed urban land expansion, morphology evolution (Chen et al., 2016a), and the evolution of land use structure by examining expansion strength (Xu and Min, 2013), compactness (Yin et al., 2010), fractal dimension (Liu et al., 2016), shape index (Wang et al., 2015), gravity-center model (Maimaiti et al., 2017; Yang et al., 2018), landscape index (Quan et al., 2015; Triantakonstantis and Stathakis, 2015), equivalent fan analysis (Zhang et al., 2016), transfer matrix (Kumar and Tripathi, 2014) etc. from a two-dimensional perspective. However, urban spatial expansion is reflected not only in the two-dimensional growth of the city but also in the increase of its vertical height. In recent years, in China's economically developed areas, with the rapid expansion of cities land space has been very limited. This requires that urban spatial expansion be changed from being extensive to being intensive, focusing on improving land use intensity in urban built-up areas (Liu et al., 2014). Therefore, the trend of three-dimensional spatial expansion in large and medium-sized cities has become increasingly discernible (Liu and Chen, 2001; Gruen, 1998). Statistically, by the end of 2012, China included 5 of the top 10 cities in the world with the highest number of high-rise buildings over $24 \mathrm{~m}$ ( 8 floors). From the perspective of the spatial organization of urban land use, urban space is three-dimensional, and three-dimensional spatial expansion is mainly reflected in the increase of building height and land plot ratio (Sun et al., 2002). However, despite the constant increase of urban building heights and the increasing contribution of vertical extension to urban volume growth, most studies have only analyzed urban morphology evolution and spatial expansion from a two-dimensional perspective, ignoring the city's vertical stretch and land use intensity change. This, to a certain extent, affects the understanding of the evolution of the urban spatial form as well as land use change laws. To comprehensively grasp the laws of urban spatial expansion and to promote scientific decision-making for urban planning and urban land use management, this paper considers the urban land use system as a whole, based on a multi-dimensional perspective (taking both two and three dimensions into account), and comprehensively analyzes urban spatial expansion using RS and GIS technology. To study urban expansion from a multi-dimensional perspective, multi-phase urban three-dimensional models must be constructed first. Most of the construction methods are to extract the roof profiles and building heights in different periods through high-resolution remote sensing images. Some literatures have studied the methods of extracting building height and floor area ratio information using remote sensing images (Weidner and Förstner, 1995; Seresht and Azizi, 2000; McIntosh and Krupnik, 2002), but the extraction accuracy and efficiency need to be improved. Based on the improvement of the above extraction method, this paper proposes the research method of multi-dimensional expansion of urban space (MEUS), and then conducts multi-angle study on MEUS.

As one of the central cities in the Yangtze River Delta, the urban spatial expansion of 
Nanjing is typical and representative (Wang et al., 2007; Chen et al., 2016b): In terms of two-dimensional spatial expansion, the proportion of urban land use in Nanjing increased rapidly after the economic reform, while the development and construction of peripheral towns gradually accelerated, and the scale of development groups continued to increase. At present, the main city and the surrounding development groups have been fully connected. Nanjing has formed a development pattern that includes one main city and three minor cities (Dongshan, Xianlin and Jiangbei), and the planar expansion of its urban built-up area is obvious. Regarding three-dimensional spatial expansion, the number of high-rise buildings in Nanjing has increased at a terrific rate since the reform. At present, the number of high-rise buildings in Nanjing ranks 19th in the world and 8th in China. This study on the expansion of urban built-up areas from a multi-dimensional perspective is therefore meaningful and can help in the discussion of MEUS research methods and enable the comprehensive understanding of MEUS laws of Nanjing.

\section{Materials and methods}

\subsection{Study area}

The research area for this study is the urban built-up area of Nanjing. Nanjing is located in southwestern Jiangsu Province, between $118^{\circ} 22^{\prime}-119^{\circ} 14^{\prime} \mathrm{E}$ and $31^{\circ} 14^{\prime}-32^{\circ} 37^{\prime} \mathrm{N}$, in one of the most important economic centers of the Yangtze River Delta. The whole city of Nanjing is composed of low hills, valley plain, lakeshore plain and riverside terrain. The Yangtze River passes through the central area of Nanjing, and the tributary of the Yangtze River, Qinhuai River and Chuhe River also flow through the city. The location and natural conditions give Nanjing a unique and superior geographical environment. In 2012, there were 11 districts and two counties in Nanjing. The research area includes contiguous built-up areas in the municipal districts, and non-contiguous built-up areas that provide important functions to the city. This includes the main city and the three minor cities, Dongshan, Xianlin, Jiangbei, as well as Banqiao, Binjiang, Lukou, Qiaolin and other new cities, and peripheral development zones, ports and airports that supply important functions to the city (Figure 1). The total study area is approximately $960 \mathrm{~km}^{2}$.

\subsection{Data sources}

To study the MEUS of Nanjing, this study primarily uses four periods of remote sensing data in 2000, 2004, 2008 and 2012. TM/ETM+ images, downloaded from the U.S. Geological Survey website (http://www.usgs.gov), are used to extract urban built-up area boundaries. Land-use maps are used to assist in extracting the boundaries of urban built-up areas. The resolutions of the TM multi-spectral and panchromatic images are $30 \mathrm{~m}$ and $15 \mathrm{~m}$, respectively. To study the MEUS of Nanjing, it is necessary to extract the outlines and heights of the buildings in urban built-up areas. To ensure the accuracy of the extraction results, high-resolution images are used in this study (Cheng and Thiel, 1995; Seresht and Azizi, 2000; Gao et al., 2008; Liu et al., 2009; Shi et al., 2009), including IKONOS and GeoEye-1 images in 2000, 2004, 2008 and 2012. The resolutions of IKONOS images are $4 \mathrm{~m}$ and $1 \mathrm{~m}$, the resolutions of GeoEye-1 images are $1.65 \mathrm{~m}$ and $0.41 \mathrm{~m}$. Due to the large scope of the study, large-scale (1:500) topographic maps, cadastral maps (1:500), and high-resolution 


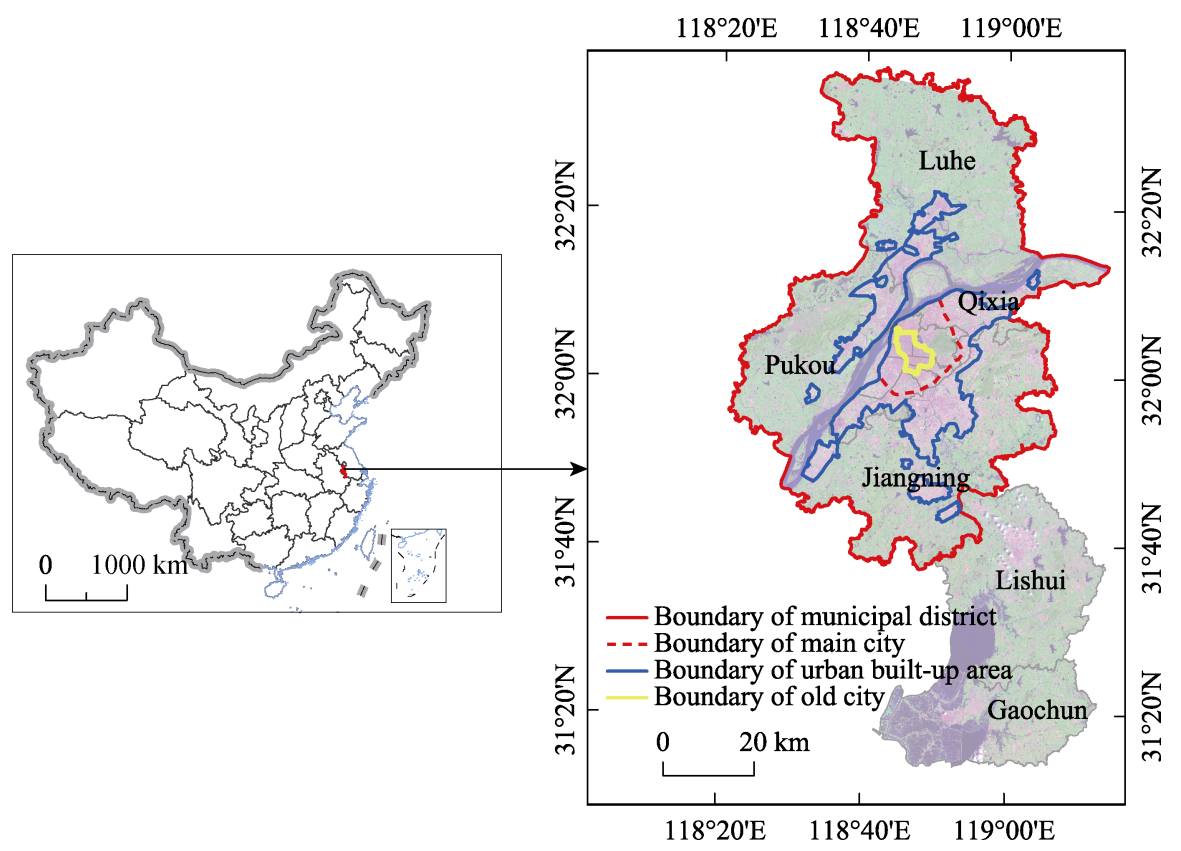

Figure 1 Map of Nanjing, Jiangsu Province, China

historical images of Google Earth are used to assist in the interpretation of regions for which lacking high-resolution images.

\subsection{Research methodology}

As shown in Figure 2, the study uses four periods of TM/ETM+ images, accurately classifies remote sensing images using the hierarchical information extraction method based on knowledge and rules (Qiao et al., 2015b) and then extracts the urban built-up area boundaries based on the classification results (Song et al., 2006; Wang and Liu, 2011). The built-up area boundaries can then be used as the boundary control of the three-dimensional information extraction and can also be used to extract the urban planar scale. The three-dimensional model is constructed by using high-resolution remote sensing images such as IKONOS, GeoEye-1 and Google Earth to extract height and roof profiles of the buildings in built-up areas for each period within the boundary of each urban built-up area. The extraction of the building roof profiles uses the object-oriented information extraction method in combination with artificial interpretation (Tan, 2010), while the extraction of building heights uses the height extraction method that's based on the typical line. The relative error of building height extracted in this paper ranges from $0.01 \%$ to $7.3 \%$ with an average error of $0.77 \mathrm{~m}$ (Qiao et al., 2015a). In regions lacking high-resolution images, large-scale topographic maps and cadastral maps are used to supplement the information sources, and finally, urban three-dimensional models for multiple periods are created (Qiao, 2013). The urban three-dimensional model constructed in this way has the characteristics of LOD1 level in detail. Details of the construction methods of the urban multi-period 3D model and the accuracies of the land use classification and building height extraction were published in an earlier series of articles for this study (Qiao et al., 2015a, 2015b). 


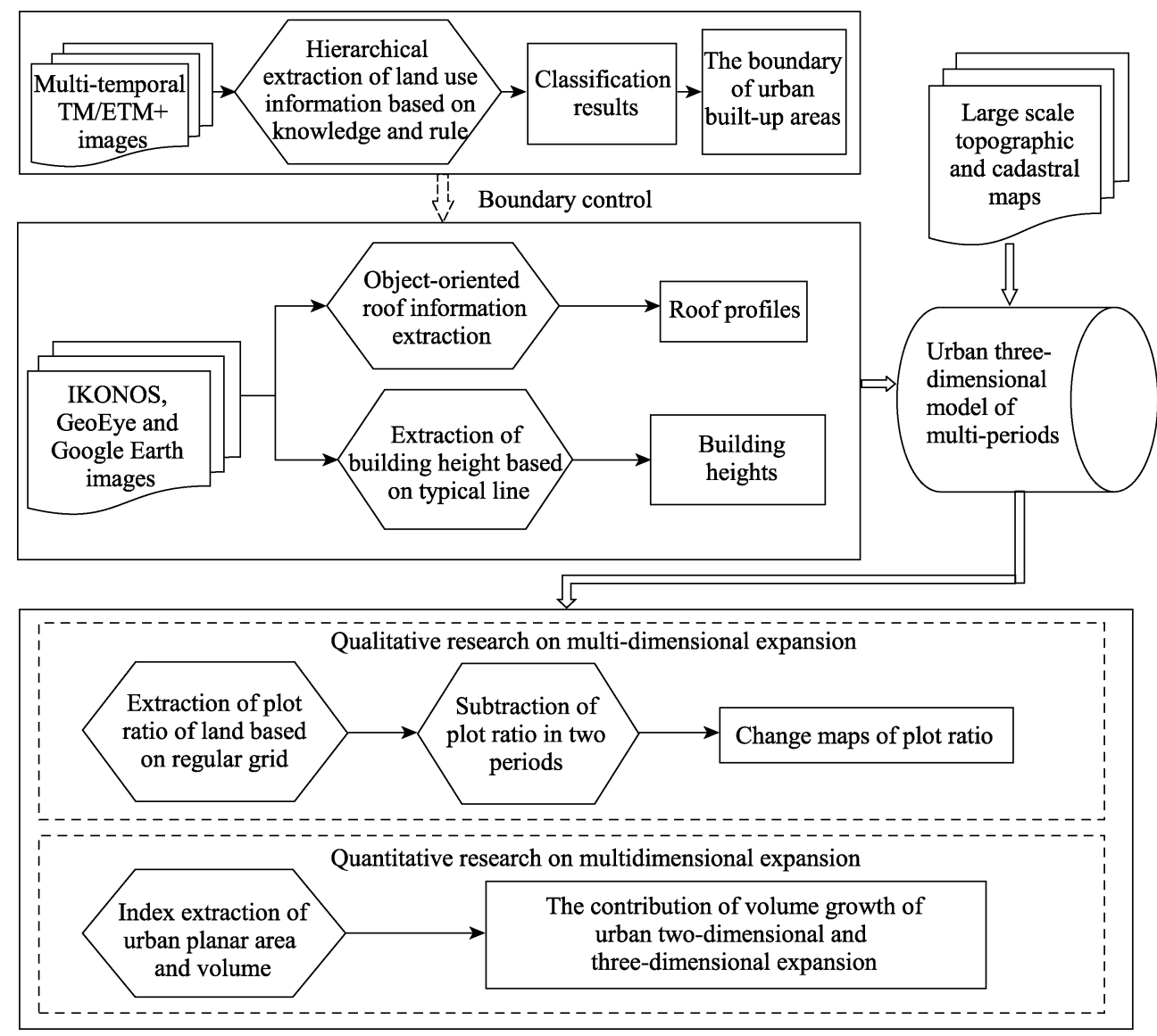

Figure 2 Technical flowchart of urban spatial multi-dimensional expansion research

This study conducts qualitative and quantitative research on urban multi-dimensional expansion based on urban three-dimensional models of multiple periods. The research divides the urban built-up areas of each stage into regular grids of a particular size and then calculates the plot ratio of each grid using the partitions statistical method in ArcGIS. Subtraction algorithms are used on the plot ratios of two adjacent grids to calculate changes in the plot ratios in each grid, allowing us to qualitatively analyze regional and magnitude changes of plot ratios. Additionally, the planar area and total urban volume during each period are calculated based on the urban three-dimensional model for each stage, and the planar area growth and volume growth for each period are then calculated. Subsequently, the urban volume growth area can be divided into two facets, one being the increasing height of buildings and the internal tapping that leads to volume growth, while the other is the epitaxial expansion and the new buildings that leading to volume growth. The percentage that each aspect contributes to the total volume growth is called the contribution rate of volume growth, and we can judge characteristics on urban multi-dimensional expansion for each period by calculating the contribution rate of volume growth. 


\section{Analysis of urban spatial multi-dimensional expansion in Nanjing}

\subsection{Generation of plot ratio change maps in urban built-up areas for each period}

According to the technique design in Figure 2, four periods of urban three-dimensional models in 2000, 2004, 2008 and 2012 are established. Based on this, the plot ratio change maps in urban built-up areas for each period are generated. This paper proposed to study changes in plot ratio based on a regular grid pattern. To determine the grid size, we performed prospective tests using ten grid size options ranging from $100 \mathrm{~m}$ to $1000 \mathrm{~m}$, with intervals of $100 \mathrm{~m}$. We found that in the context of Nanjing urban built-up areas, computation is moderate with grids of $500 \mathrm{~m}$ and that size well reflects the plot ratio change laws in the entire urban built-up area. Some buildings fall in multiple adjacent grids due to a regular grid pattern being used as the computational element. To ensure statistical accuracy of the building volume within the computational element, we performed overlay analysis on the three-dimensional model and the regular grid for each period in the research area before executing the statistics and split any building that fell between adjacent grids into two or more buildings. We calculated the volume of each building and the average plot ratio of each grid, in combination with the height of buildings based on the calculation of the floor space of each building within the grid. We performed subtraction algorithms on the average plot ratio for two adjacent grids of each period (by subtracting the previous period from the latter period) using GIS spatial overlay analysis (Han et al., 2005). Then, we generated a chart of plot ratio changes in urban built-up areas for the three periods (2000-2004, 2004-2008 and 2008-2012) according to the statistics of the regular grid (Li et al., 2007; Zha, 2011). The diagram has boundary lines of the urban built-up areas for each initial period. Therefore, two situations are reflected within the line; one is the internal tapping of construction land, and the other is the reconstruction of the old city leading to the increase of urban spatial expansion. The situation of urban expansion leading to an increase of urban space is reflected beyond the line (Li, 2007). A small amount of grid data may be negative after subtracting, mainly due to the removal of old buildings in the grid during that period, with new buildings having not yet been built by the end of the period. Using the groupings of $\leqslant 0,0-0.25$, $0.25-5,0.25-0.75,0.75-1$ and $>1$, we divide the plot ratio variations of each grid into six levels and represented them in different colors. The plot ratio change maps are shown in Figure 3.

\subsection{Analysis of plot ratio changes in Nanjing urban built-up areas}

Because the boundary of the urban built-up area in the initial period is marked on the plot ratio change map, we can analyze the two-dimensional and three-dimensional expansions of the urban built-up area at the same time. The planar expansion and the expansion intensity are reflected outside of the boundary, and the plot ratio change in the urban built-up area is reflected within the boundary.

(1) Plot ratio changes from 2000 to 2004 . The planar expansion of urban space was concentrated mainly in the south and southeast of the city, including the core area of Xianlin University City, most regions of Qilin town, the western regions of Refinery, south and east of the Jiangning district, the pre-construction of the Olympic New City, northeast of Nanjing 
Chemical Industrial Park, the Luhe Development Zone, Nanjing University of Information Science and Technology and its surroundings, etc. For plot ratio changes in built-up areas, the grid plot ratio along both sides of the main roads within the city grew rapidly. The plot ratio of the Hexi area in the Gulou District displayed fast growth. The plot ratio of the Dachang area north of the Yangtze River and the old town in Luhe also grew rapidly and were the main areas of urban construction land reconstruction and internal potential development (IPD) in Nanjing for the four years from 2000 to 2004. For plot ratio changes in new expansion areas of the city, the plot ratio of added residential areas in Jiangning and Xianlin were large, and more grids with values over 1 are generated in these areas. However, for the rest of the newly added construction area, the plot ratio is general, with most grids having average values below 0.5 (Figure $3 a$ ).

(2) Plot ratio changes from 2004 to 2008. During this period, the planar expansion of urban space was in the east, north and west of the Xianlin core area, the Shangfang Industrial Park, east and south of Jiangning, Lukou New City, the Yuhua Development Zone, the Binjiang Development Zone of Jiangning, etc. The planar expansion speed was fast during this period as a large area of cultivated land around the city occupied. The overall performance showed that radial expansion was evident. From the view of plot ratio changes within the built-up areas, changes were large along both sides of the main roads, as well as the Hexi area and east and north of the main urban area. Overall, the IPD intensity was somewhat low. There was a scattered distribution of grids with great changes in plot ratio, which reflects that the pace of reconstruction and IPD in this city was slowing down from 2004 to 2008, integration of elements was not concentrated, the scale was small, and urban transformation was sporadic. For the newly built-up areas, the plot ratios were not very high compared to the period from 2000 to 2004. Higher plot ratio existed only in some residential areas (Figure $3 b)$.

(3) Plot ratio changes from 2008 to 2012. The planar expansion of urban space was mainly reflected in the construction of the development zones of Luhe, Pukou and other areas, the eastward and southward expansion of Xiongzhou town in Luhe, the construction of the southern area of the Binjiang Development Zone, etc. During this period, only a small amount of planar expansion occurred in Jiangning, Xianlin. Urban expansion and the construction of development zones were the main causes for planar expansion. The construction of development zones was mostly in the form of infrastructure construction, road network had been basically formed and standard workshops were built in some areas. The new urban construction land was used to build regular multi-story residential areas and the land use intensity in these areas was relatively high. The plot ratio change characteristics in urban built-up areas were development and construction near the Nanjing South Railway Station and the Olympic New City from 2008 to 2012, which significantly increased the urban three-dimensional space of these two locations. Most high-rise buildings that were built on commercial finance land along the Zhongshan East Road, the Zhongshan Road, the Zhongshan North Road and other roads within the main urban area, helped to increase the urban three-dimensional space of these areas. In addition, the development and construction of high-rise and multi-story residential areas in Jiangning, Xianlin, Luhe and Pukou also helped to improve the plot ratios in these areas. Urban sprawl slowed, but IPD significantly sped up. The areas in which plot ratio increased were more concentrated and had a clustered distribu- 
tion with larger areas. This reflected an obvious increase in the contribution rate of three-dimensional spatial expansion for urban spatial expansion (Figure 3c).

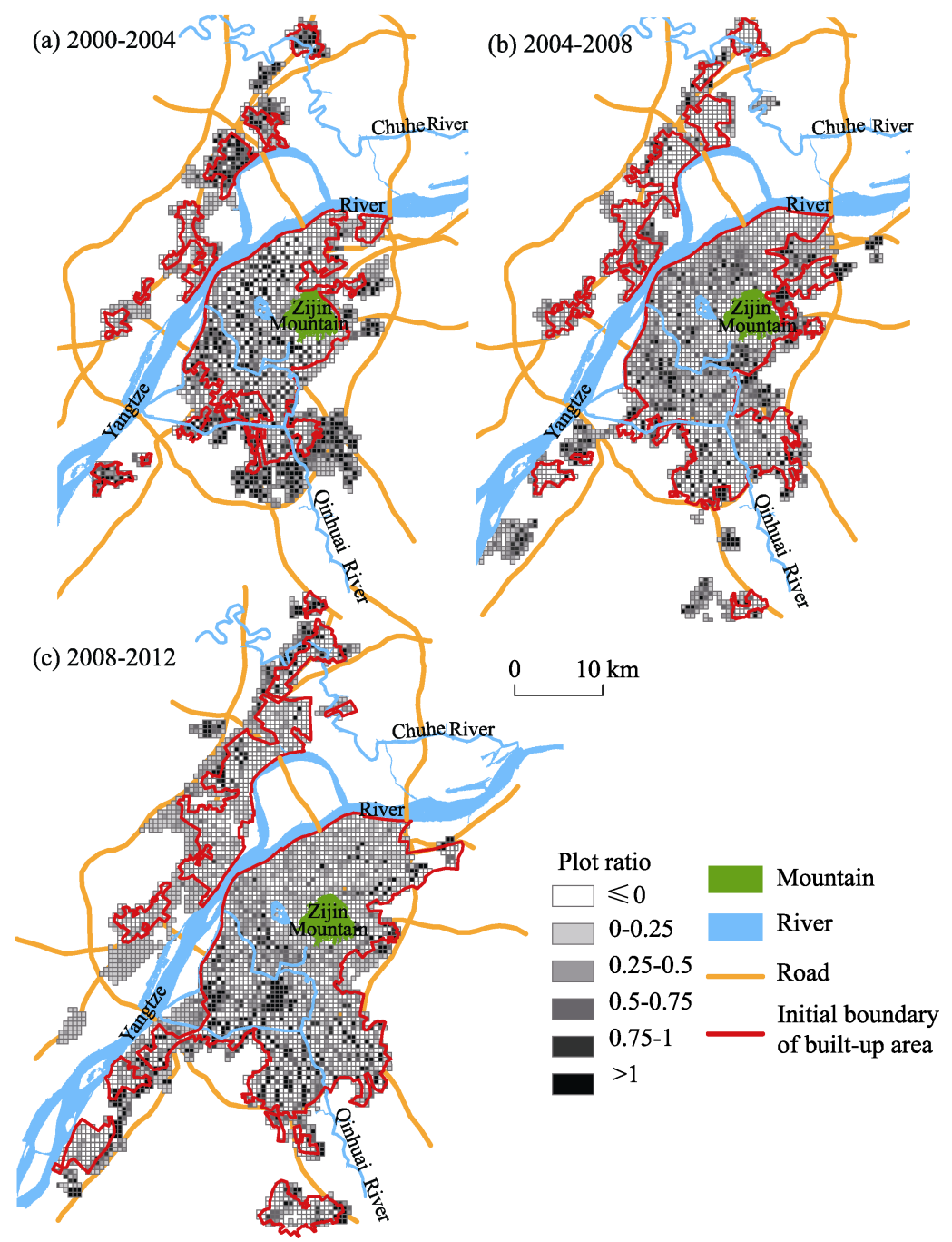

Figure 3 Staged variation maps of land use expansion and plot ratio change in Nanjing for 2000-2004 (a), 2004-2008 (b) and 2008-2012 (c)

\subsection{Analysis of the quantitative characteristics of MEUS}

In the process of urban spatial growth, planar expansion is always accompanied by growth in three-dimensional space. One aspect of this growth is the expansion of the planar scale, while another is the filling in of the original urban internal space through the increase in building heights and plot ratios. Using four periods of urban three-dimensional models along with the geostatistical method, urban built-up area, urban volume and other data for different periods are calculated. Followed by the calculation of the urban planar expansion area, the amount of volume growth, the amount of IPD volume growth, the amount of planar expan- 
sion volume growth and its corresponding contribution rate of volume growth are extracted during each period. In order to better analyze the relationship between urban two-dimensional expansion and three-dimensional expansion, as many indicators as possible are selected to analyze urban multi-dimensional expansion. It is of great significance to grasp the characteristics of urban expansion during each period, and can also provide a basis for urban planning management and urban land use. The statistical data are shown in Table 1.

Table 1 Volume growth contribution of two-dimensional and three-dimensional urban spatial expansion in Nanjing

\begin{tabular}{|c|c|c|c|c|}
\hline Serial No. & Index & $2000-2004$ & 2004-2008 & $2008-2012$ \\
\hline (1) & Area of the city at the beginning of the period $\left(\mathrm{km}^{2}\right)$ & 354.10 & 533.37 & 754.50 \\
\hline (2) & Area of the city at the end of the period $\left(\mathrm{km}^{2}\right)$ & 533.37 & 754.50 & 960.08 \\
\hline (3) & Urban planar expansion area $\left(\mathrm{km}^{2}\right)$ & 179.27 & 221.13 & 205.58 \\
\hline (4) & Growth rate of urban planar area (\%) & 50.63 & 41.46 & 27.25 \\
\hline$(5)$ & Total urban volume at the beginning of the period $\left(\mathrm{m}^{3}\right)$ & $9.19 \times 10^{8}$ & $1.39 \times 10^{9}$ & $1.70 \times 10^{9}$ \\
\hline (6) & Total urban volume at the end of the period $\left(\mathrm{m}^{3}\right)$ & $1.39 \times 10^{9}$ & $1.70 \times 10^{9}$ & $2.07 \times 10^{9}$ \\
\hline (7) & $\begin{array}{l}\text { Total volume at the end of the period within the urban } \\
\text { planar scope at the end of the period }\left(\mathrm{m}^{3}\right)\end{array}$ & $1.02 \times 10^{9}$ & $1.46 \times 10^{9}$ & $1.97 \times 10^{9}$ \\
\hline (8) & Volume growth of urban IPD $\left(\mathrm{m}^{3}\right)$ & $1.04 \times 10^{8}$ & $7.59 \times 10^{7}$ & $2.72 \times 10^{8}$ \\
\hline (9) & Volume growth of urban planar expansion $\left(\mathrm{m}^{3}\right)$ & $3.64 \times 10^{8}$ & $2.34 \times 10^{8}$ & $9.88 \times 10^{7}$ \\
\hline$(10)$ & Volume growth of IPD per unit area $\left(\mathrm{m}^{3} / \mathrm{km}^{2}\right)$ & $2.94 \times 10^{5}$ & $1.42 \times 10^{5}$ & $3.61 \times 10^{5}$ \\
\hline (11) & Total amount of urban volume growth $\left(\mathrm{m}^{3}\right)$ & $4.69 \times 10^{8}$ & $3.10 \times 10^{8}$ & $3.71 \times 10^{8}$ \\
\hline$(12)$ & Growth rate of total urban volume $(\%)$ & 50.97 & 22.32 & 21.87 \\
\hline (13) & Contribution rate of volume growth of urban expansion (\%) & 77.79 & 75.49 & 26.62 \\
\hline (14) & Contribution rate of volume growth of urban IPD (\%) & 22.21 & 24.51 & 73.38 \\
\hline
\end{tabular}

Note: $(4)=((2)-(1)) /(1) ;(8)=(7)-(5) ;(9)=(6)-(7) ;(10)=(8) /(5) ;(12)=((6)-(5)) / 5 ;(13)=(9) /(11) ;(14)=(8) /(11)$

Table 1 shows that the growth rates of the planar area for the three periods were $50.63 \%$, $41.46 \%$ and 27.25\%. During 2008-2012, the planar growth rate had a significant reduction. The volume growth rates for the three periods were $50.97 \%, 22.32 \%$ and $21.87 \%$. The volume growth rates for the last two periods were significantly lower than the first period, but the difference between the last two periods was small. The ratios between the planar area growth rate and the urban volume growth rate were $1.01,0.54$ and 0.80 respectively. The intensity degree of urban spatial growth changed from relatively high to low and then gradually rose.

Based on the data in Table 1, the quantitative characteristics of MEUS in each stage can be determined and are as follows:

(1) 2000-2004: Planar expansion plays the main role, IPD intensity was large, but the contribution rate was not high. During this period, urban planar expansion is in the accelerating stage and the annual average expansion is $44.82 \mathrm{~km}^{2}$. IPD intensity is high, and the volume growth of IPD per unit area is $2.94 \times 10^{5} \mathrm{~m}^{3} / \mathrm{km}^{2}$. Urban planar expansion was mainly reflected in the construction of residential areas and the core area of University City. In the expansion area, the plot ratio is relatively high. MEUS had two characteristics at this stage. On the one hand, urban sprawl is relatively fast, the built-up area is growing rapidly, and at 
the same time, the utilization efficiency and expansion intensity are higher. On the other hand, the scale of IPD is larger, and planar expansion and IPD were carried out at the same time. IPD intensity was high but the contribution rate of volume growth was not high due to the volume growth of planar expansion also being large. The contribution rate was only $22.21 \%$.

(2) 2004-2008: Planar expansion accelerated, and IPD intensity slowed down. During this period, urban planar expansion was at the high-speed expansion stage, and the annual average expansion was $55.28 \mathrm{~km}^{2}$. The volume growth of IPD per unit area was just $1.42 \times 10^{5}$ $\mathrm{m}^{3} / \mathrm{km}^{2}$, and the strength was less than half of that in 2000-2004. The main performance of urban space growth was the volume growth resulting from planar expansion. The IPD of urban built-up area was weak. The speed of urban planar expansion was fast during this period, and the expansion mode of urban construction land was relatively rough and simple. The utilization intensity of new construction land decreased significantly compared with that of the previous period, and the reasons were as follows: On the one hand, the area of urban planar expansion increased rapidly in a short time; on the other hand, the construction of the industrial park was the main cause of the expansion in this period and the plot ratio was low. At the same time, MEUS in Nanjing was unbalanced due to the low intensity and scale of IPD, and urban volume growth depended mainly on the volume growth of the new built-up areas.

(3) 2008-2012: Planar expansion slowed down, and IPD intensity increased. The speed of urban planar expansion slowed down during 2008-2012, and the annual average expansion was $51.40 \mathrm{~km}^{2}$. The volume growth of IPD per unit area was $3.61 \times 10^{5} \mathrm{~m}^{3} / \mathrm{km}^{2}$; this is the highest level for the three periods, and the intensity increases obviously compared to the intensity in 2004-2008. The volume growth contribution rate of urban planar expansion only accounted for $26.62 \%$ of the total volume growth because most of the buildings within the expanded section had not been completed. The effect of IPD was obvious during this period, the volume growth of IPD was up to $2.72 \times 10^{8} \mathrm{~m}^{3}$. The volume growth of IPD and the volume growth of IPD per unit area reached the highest levels in history, which reflected that Nanjing paid greater attention to the adjustment and optimization of the structure of inner urban construction land from 2008 to 2012. Making urban IPD an important means of urban spatial growth (Zhao et al., 2010).

\section{Conclusions and discussion}

\subsection{Conclusions}

(1) This paper builds multi-temporal urban three-dimensional models using remote sensing and GIS technology. On the basis of the three-dimensional models, this study extracts urban land plot ratios by means of zonal statistics based on regular grids and then qualitatively analyzes the region and magnitude of urban two-dimensional and three-dimensional expansion through plot ratio change maps, which are generated using the overlay analysis method. In addition, based on the urban three-dimensional model, this study uses the geostatistical method to analyze the numerical characteristics of MEUS and quantitatively analyzes the characteristics of MEUS in Nanjing for different stages based on the concept of the volume 
growth contribution rate. The method adopted in this paper can effectively analyze the law and characteristics of MEUS, and it has applicability to MEUS research in other regions. At the same time, it has good application potential in urban planning, land management, smart city construction, and disaster prevention and mitigation.

(2) Taking Nanjing as an example, this paper qualitatively and quantitatively analyzes the law of MEUS since the new century in 2000-2004, 2000-2008 and 2008-2012. The results show the following: (i) During 2000-2004, planar expansion played the main role, and IPD intensity was high with lower volume growth contribution rate. The planar space expansion was mainly concentrated in the south and southeast of the city. Aside from the residential plot ratio in the newly constructed areas of Jiangning and Xianlin being large, the plot ratio for the rest of the newly construction area was average. (ii) During 2004-2008, planar expansion accelerated, and IPD intensity slowed down. The volume growth of IPD per unit area was only $1.42 \times 10^{5} \mathrm{~m}^{3} / \mathrm{km}^{2}$, less than half of the previous stage. The urban planar expansion occupied a large portion of cultivated land around the city. Generally, the urban expansion occurred through distinct radial expansion. At the same time, the grids whose plot ratio changed greatly were dispersedly distributed, the scale of IPD was small and had sporadic transformation. Compared with the former stage, the plot ratio of the newly expanded area was not very high. (iii) During 2008-2012, planar expansion slowed down, and IPD intensity was greater. The volume growth of IPD per unit area was $3.61 \times 10^{5} \mathrm{~m}^{3} / \mathrm{km}^{2}$, which was the highest level of the three periods. Urban expansion and development zone construction held a large proportion in the planar expansion, and the speed of urban planar expansion slowed down. The regions whose plot ratios increased in the built-up area were relatively concentrated, with clustered distribution and large areas, and the contribution rate of the three-dimensional spatial expansion to urban spatial expansion obviously increased.

(3) In the three periods, the average annual urban planar expansion was $44.82 \mathrm{~km}^{2}, 55.28$ $\mathrm{km}^{2}$ and $51.40 \mathrm{~km}^{2}$, and the average annual volume growth was $1.17 \times 10^{8} \mathrm{~m}^{3}, 7.75 \times 10^{7} \mathrm{~m}^{3}$ and $9.28 \times 10^{7} \mathrm{~m}^{3}$. The ratio between the growth rate of the planar area and the growth rate of urban volume was $1.01,0.54$ and 0.80 , which indicated that the intensive degree of urban spatial growth changed from relatively high to low, then rose gradually. The contribution rate of volume growth to urban IPD during the three periods was $22.21 \%, 24.51 \%$ and $73.38 \%$, which reflected that Nanjing began to pay more attention to the adjustment and optimization of the structure of urban inner construction land; urban renewal efforts continued to strengthen in the third stage.

\subsection{Discussion}

This paper provides a beneficial discussion on the research method of MEUS. However, because the paper mainly adopts the use of remote sensing images for the construction of urban three-dimensional models, we failed to take into account the expansion of underground space. In this paper, the urban three-dimensional model only extracted roof profile and building height, but for a large portion of the urban built-up area, the workload of the construction of a multi-temporal urban three-dimensional model is still huge, so improving the automation level of modeling will be an urgent need to be addressed in future research. 


\section{References}

Anas A, 1976. Short-run dynamics in the spatial housing market. In: Papageorgiou G J (eds.). Mathematical Land Use Theory. Lexington: Lexington Books, 262-275.

Chen J L, Gao J L, Yuan F, 2016a. Growth type and functional trajectories: An empirical study of urban expansion in Nanjing, China. PLoS ONE, 11(2): e0148389. doi: 10.1371/journal.pone.0148389.

Chen J L, Gao J L, Yuan F et al., 2016b. Spatial determinants of urban land expansion in globalizing Nanjing, China. Sustainability, 8(9): 868. doi: 10.3390/su8090868.

Cheng F, Thiel K H, 1995. Delimiting the building heights in a city from the shadow in a panchromatic SPOT-image: Part 1. Test of forty-two buildings. Remote Sensing, 16(3): 409-415. doi: 10.1080/01431 169508954409.

Chi W F, Shi W J, Kuang W H, 2015. Spatio-temporal characteristics of intra-urban land cover in the cities of China and USA from 1978 to 2010. Journal of Geographical Sciences, 25(1): 3-18. doi: 10.1007/s11442-015$1149-\mathrm{z}$.

Gao X, Zhao D L, Zhang W, 2008. On the methods of obtaining the building height information from high-resolution remote sensing images. Bulletin of Surveying and Mapping, (3): 41-43. (in Chinese)

Gruen A, 1998. TOBAGO: A semi-automated approach for the generation of 3D building models. ISPRS Journal of Photogrammetry \& Remote Sensing, 53(2): 108-118. doi: 10.1016/S0924-2716(97)00034-8.

Han X P, Xu J G, Fu X M, 2005. A study on estimating urban FAR based on high-resolution satellite images. Remote Sensing Information, (2): 24-28. (in Chinese)

Kumar M, Tripathi D K, 2014. Spatial monitoring of urban growth of Nagpur City (India) using geospatial techniques. Journal of Settlements \& Spatial Planning, 5(2): 91-98.

Li F X, 2007. Research on urban expansion information tupu of Nanjing [D]. Nanjing: Nanjing University. (in Chinese)

Li J Y, Zhang L, Wu B F et al., 2007. Study on extracting building density and floor area ratio based on high resolution image. Remote Sensing Technology and Application, 22(3): 309-313. (in Chinese)

Liu F, Zhang Z X, Shi L F et al., 2016. Urban expansion in China and its spatial-temporal differences over the past four decades. Journal of Geographical Sciences, 26(10): 1477-1496. doi: 10.1007/s11442-016-1339-3.

Liu L F, Wang R J, Dong W P et al., 2009. A fast method extracting building height using high resolution satellite image. Remote Sensing Technology and Application, 24(5): 631-634. (in Chinese)

Liu M H, Chen Y G, 2001. Experimental methods of geographical researches: From modeling material objects to computer simulation. Journal of Xinyang Teachers College (Natural Science Edition), 24(1): 209-213. (in Chinese)

Liu Y S, Fang F, Li Y H, 2014. Key issues of land use in China and implications for policy making. Land Use Policy, 40(4): 6-12.

Liu Y S, Li Y H, 2017. Revitalize the world's countryside. Nature, 548(7667): 275-277.

Maimaiti B, Ding J L, Simayi Z, 2017. Characterizing urban expansion of Korla City and its spatial-temporal patterns using remote sensing and GIS methods. Journal of Arid Land, 9(3): 458-470.

McIntosh K, Krupnik A, 2002. Integration of laser-derived DSMs and matched image edges for generating an accurate surface model. ISPRS Journal of Photogrammetry and Remote Sensing, 56(3): 167-176.

Qiao W F, 2013. Study on urban spatial multidimensional expansion of Nanjing based on land use perspective [D]. Nanjing: Nanjing Normal University. (in Chinese)

Qiao W F, Liu Y S, Xiang L Z et al., 2015a. Research on extracting building height rapidly based on high-resolution remote sensing images without parameters. Journal of Geo-information Science, 17(8): 995-1000. (in Chinese)

Qiao W F, Wang Y H, Xiang L Z, 2015b. Hierarchical extraction of land use information based on knowledge and rule. Resources and Environment in the Yangtze Basin, 24(7): 1079-1085. (in Chinese)

Quan B, Bai Y J, Römkens M J M et al., 2015. Urban land expansion in Quanzhou city, China, 1995-2010. Habitat International, 48: 131-139. doi: 10.1016/j.habitatint.2015.03.021. 
Seresht M S, Azizi A, 2000. Automatic building recognition from digital aerial images. International Archives of Photogrammetry and Remote Sensing, 33(B3/2; PART 3): 792-798.

Shi L Y, Shao G F, Cui S H et al., 2009. Urban three-dimensional expansion and its driving forces: A case study of Shanghai, China. Chinese Geographical Science, 19(4): 291-298. doi: 10.1007/s11769-009-0291-x.

Song X D, Liu P, Zhou Y X, 2006. Urban and rural area division: Taking Shanghai as an example. Acta Geographica Sinica, 61(8): 787-797. (in Chinese)

Sun M, Ma A N, Chen J, 2002. Review on three-dimensional city model research. Journal of Remote Sensing, 6(2): 155-160. ( in Chinese)

Tan Q L, 2010. Urban building extraction from VHR multi-spectral images using object-based classification. Acta Cartographica Sinica, 39(6): 618-623. (in Chinese)

Triantakonstantis D, Stathakis D, 2015. Examining urban sprawl in Europe using spatial metrics. Geocarto International, 30(10): 1092-1112. doi: 10.1080/10106049.2015.1027289.

United Nations (UN), 2008. World Urbanization Prospects: The 2007 Revision. New York: United Nations, 3-4.

Wang L, Li C C, Ying Q et al., 2012. China's urban expansion from 1990 to 2010 determined with satellite remote sensing. Chinese Science Bulletin, 57(22): 2802-2812. doi: 10.1007/s11434-012-5235-7. (in Chinese)

Wang J, Liu J, 2011. Dynamic of urban expansion in Lianyungang city based on the multi-time-phase remote sensing images: A case study of Xinpu District. Urban Geotechnical Investigation \& Surveying, 1: 81-83. (in Chinese)

Wang Q, Zhang Z X, Yi L et al., 2007. Research on urban expansion in Nanjing, China using RS and GIS. Resources and Environment in the Yangtze Basin, 16(5): 554-559. (in Chinese)

Wang X S, Liu J Y, Zhuang D F et al., 2005. Spatial-temporal changes of urban spatial morphology in China. Acta Geographica Sinica, 60(3): 392-400. (in Chinese)

Weidner U, Förstner W, 1995. Towards automatic building extraction from high-resolution digital elevation models. ISPRS Journal of Photogrammetry and Remote Sensing, 50(4): 38-49.

Wen Q K, Zhang Z X, Shi L F et al., 2016. Extraction of basic trends of urban expansion in China over past 40 years from satellite images. Chinese Geographical Science, 26(2): 129-142. doi: 10.1007/s11769-016-0796-z.

$\mathrm{Xu}$ X L, Min X B, 2013. Quantifying spatiotemporal patterns of urban expansion in China using remote sensing data. Cities, 35: 104-113. doi: 10.1016/j.cities.2013.05.002.

Yang Y Y, Liu Y S, Li Y R et al., 2018. Quantifying spatio-temporal patterns of urban expansion in Beijing during 1985-2013 with rural-urban development transformation. Land Use Policy, 74(5): 220-230.

Yin F, Feng M, Zhong F et al., 2010. Research of urban expansion in Siping city based on remote sensing and GIS. Journal of Geo-Information Science, 12(2): 242-247. (in Chinese)

Zha Y, 2011. A study on remote sensing methods in estimating urban build-up volume ratio based on aerial photographs. Progress in Geography, 20(4): 378-383. (in Chinese)

Zhao S Q, Zhou D C, Zhu C et al., 2015. Spatial and temporal dimensions of urban expansion in China. Environmental Science \& Technology, 49(16): 9600-9609. doi: 10.1021/acs.est.5b00065.

Zhao X F, Huang X J, Chen Y et al., 2010. Research progress in urban land intensive use. Journal of Natural Resources, 25(1): 1979-1996. (in Chinese)

Zhang Z X, Li N, Wang X A et al., 2016. Comparative study of urban expansion in Beijing, Tianjin and Tangshan from the 1970s to 2013. Remote Sensing, 8(6): 496. doi: 10.3390/rs8060496. 